\title{
Pancreatic Intraductal Papillary-Mucinous Neoplasm with an Associated Invasive Carcinoma
}

National Cancer Institute

\section{Source}

National Cancer Institute. Pancreatic Intraductal Papillary-Mucinous Neoplasm with an Associated Invasive Carcinoma. NCI Thesaurus. Code C5726.

A pancreatic intraductal papillary mucinous neoplasm characterized by the presence of a focal or multifocal invasive carcinomatous component. The invasive carcinoma is either colloid or ductal adenocarcinoma. 